A 15 year prospective study of 18 children with benign childhood epilepsy with occipital paroxysms was carried out at the Division of Neurology and Clinical Neurophysiology, King Khalid University Hospital, Riyadh, Saudi Arabia. These patients represented one-fifth of all benign age-and localization-related idiopathic epilepsies seen with onset before the age of 13 years. There was a preponderance in females and peak age at onset was five years. The seizures consisted of tonic deviation of the eyes and vomiting, followed by unilateral or generalized convulsions. They were mainly nocturnal. Remission usually occurred one to two years after onset and no seizures occurred after 12 years of age. Two children were exceptional, having frequent diurnal episodes with visual hallucinations, postictal headache, and occasional nocturnal hemiconvulsions. EEG abnormalities consisted of repetitive spike and slow-wave discharges in the occipital regions, attenuated with eyes open. The EFG abnormalities persisted after clinical remission, sametimes up to age 16. (Panayiotopoulos CP. Benign childhood epilepsy with occipital paroxysms: a 15-year prospective study. Ann Neurol July 1989; 26:51-56).

COMENT. The authors propose a definition of benign childhood epilepsy with occipital paroxysms as follows: a syndrome of brief or prolonged partial seizures marked by deviation of the eyes and vamiting. The seizures are usually nocturnal and frequently evolve to hemiconvulsions and generalized tonic-clonic fits. Onset is between the ages of two and eight years, with a peak occurring at five years and remission before 12 years. There is a preponderance in females and prognosis is excellent. In addition, there is a late onset variant with mainly diurnal seizures consisting of visual symptoms often followed by hemiclonic seizures or autamatisms and migraine headache. Prognosis appears relatively good. The EEG in both shows repetitive occipital spikes/sharp and slow waves that are often asymmetrical and which attenuate or disappear when the eyes are open.

This syndrome is related to basilar migraine with epileptiform EEG abnormalities (Camfield PR, Metrakos K, Andermann F. Neurology $1978 ; 28: 584)$.

\title{
REVISED CLASSIFICATION OF EPILEPSIES
}

The proposals for a revised classification of epilepsy and epileptic syndromes (1981-1985) have again been revised by the cammission on Classification and Terminology of the International League Against Epilepsy. The major classes are I. generalized epilepsy, and II. localization related, partial or focal epilepsies. Epilepsies of known etiology (symptomatic or secondary "epilepsies" are separated from idiopathic (primary) and cryptogenic. Idiopathic epilepsies are distinguished from cryptogenic epilepsies. Idiopathic epilepsies are defined by age related onset, clinical and electroencephalographic characteristics and a presumed genetic etiology. Cryptogenic epilepsies are presumed to be symptomatic and the etiology is unknown; they are age related but often do not have well defined electroclinic characteristics. 